\title{
Neutron production in the lunar subsurface from alpha particles in galactic cosmic rays
}

\author{
Shuya Ota ${ }^{1,2,3}$, Shingo Kobayashi ${ }^{4}$, Lembit Sihver ${ }^{3,5,6}$, Naoyuki Yamashita ${ }^{1}$, and Nobuyuki Hasebe ${ }^{1}$ \\ ${ }^{1}$ Research Institute for Science and Engineering, Waseda University, 3-4-1 Okubo, Shinjuku-ku, Tokyo, Japan \\ ${ }^{2}$ National Institute of Radiological Sciences, 4-9-1 Anagawa, Inage, Chiba, Japan \\ ${ }^{3}$ Chalmers University of Technology, Goteborg, SE-412 96 Gothenburg, Sweden \\ ${ }^{4}$ Japan Aerospace Exploration Agency, 3-1-1 Yoshinodai, Sagamihara, Kanagawa, Japan \\ ${ }^{5}$ Texas A\&M University, Texas 77843-3133, USA \\ ${ }^{6}$ Roanoke College, Salem, Virginia 24153, USA
}

(Received July 3, 2009; Revised January 19, 2010; Accepted January 22, 2010; Online published February 21, 2011)

\begin{abstract}
The neutron production from alpha particles in galactic cosmic rays (GCR) in the lunar subsurface has not been estimated with reliable precision despite its importance for lunar nuclear spectroscopy and space dosimetry. Here, we report our estimation of neutron production from GCR nuclei (protons and alpha particles) with the Particle and Heavy Ion Transport code System (PHITS), which includes several heavy ion interaction models. PHITS simulations of the equilibrium neutron density profiles in the lunar subsurface are compared with experimental data obtained in the Apollo 17 Lunar Neutron Probe Experiment. Our calculations successfully reproduced the data within an experimental error of 15\%. Our estimation of neutron production from GCR nuclei, estimated by scaling that from protons by a factor of 1.27 , is in good agreement within an error of $1 \%$ with the calculations using two different alpha particle interaction models in PHITS during a period of average activity of the solar cycle. However, we show that the factor depends on the incident GCR spectrum model used in the simulation. Therefore, we conclude that the use of heavy ion interaction models is important for estimating neutron production in the lunar subsurface.
\end{abstract}

Key words: Neutron, alpha particle, lunar and planetary spectroscopy, Moon, gamma ray spectrometer, SELENE, PHITS, heavy ion transportation.

\section{Introduction}

The lunar surface is quite distinct from Earth's surface. Since the Moon has no atmosphere and its magnetic field is very weak, the lunar surface is directly exposed to galactic cosmic rays (GCR). Secondary products, including neutrons and gamma rays, are continuously produced by the nuclear interactions between the GCR nuclei and the materials in the lunar subsurface. In the field of lunar science, it is very important to know the production rates of these secondary products because the neutrons and gamma rays emitted from the lunar surface can be used to estimate the elemental abundance of the lunar subsurface material (Evans et al., 1993; Feldman et al., 1993). The composition of the Moon's surface has been investigated using neutron and gamma ray spectroscopes following the launch of the Lunar Prospector (e.g., Gasnault et al., 2000; Lawrence et al., 2004). Above all, the recent successful mission of the Japanese lunar orbiter SELENE (KAGUYA) equipped with a Ge gamma ray spectrometer is expected to report drastically improved global mapping data of the lunar surface composition (Hasebe et al., 2008, 2009). In order to be able to derive the absolute abundance of elements by nu-

Copyright (c) The Society of Geomagnetism and Earth, Planetary and Space Sciences (SGEPSS); The Seismological Society of Japan; The Volcanological Society of Japan; The Geodetic Society of Japan; The Japanese Society for Planetary Sciences; TERRAPUB.

doi:10.5047/eps.2010.01.006 clear spectroscopy, lunar scientists need a good simulation code for transporting the GCR nuclei and secondary products in the lunar material.

From the view of not only lunar science but also human activities on the Moon, the simulation of particle production and transportation in matter is essential. Future human activities on the lunar surface will lead to further progress in space science. To this end, the building of a lunar base has been seriously discussed for a long period of time. On the lunar surface, human beings would be exposed to not only to both primary GCR and their secondary radiation from the lunar surface but also to high energetic solar energetic particles (SEPs). Consequently, the shielding of human beings from intense radiation exposure is one of major problems to be solved to secure the safety of future human activities on the lunar surface. The unshielded dose equivalent rate on the Moon is currently estimated to be $300-400 \mathrm{mSv} / \mathrm{year}$ (Adams et al., 2007), depending on the solar cycle, but it has recently been estimated to be as high $>800 \mathrm{mSv} / \mathrm{year}$ during the solar minimum of the solar cycle (Hayatsu et al., 2008, 2009); in comparison, the unshielded dose equivalent rate is about $2.4 \mathrm{mSv} /$ year on the Earth (UNSCEAR report, 1988). This variability in dose estimation mainly results from uncertainties in the observational data of GCR spectra, composition of the lunar soil, and the particle and heavy ion transportation code. As the qualities and quantities of the observational data are progressively being improved by the 
launching of continuous lunar missions, such as SELENE, Chang'e, and Chandrayaan-1, transport simulations with an increased accuracy is required.

Many transport codes have been developed for the research of fundamental high-energy particles, and these are used for radioprotection in space and for the treatment planning systems (TPSs), such as heavy ion cancer therapy. These codes typically include MCNP (Briesmeister, 1997), FLUKA (Fasso et al., 1993), Geant4 (Agostinelli et al., 2003), and PHITS (Particle and Heavy Ion Transport code System) (Niita et al., 1995, 2006). These transport codes are continuously being improved, such as the development of new and better models for nuclear and electromagnetic interactions and the extension of the energy region for the nuclear data libraries, etc. A careful comparison of these codes is found in Sihver et al. (2008a). Since each code has its own specific advantages and disadvantages, it is essential to use the best transport code for the purpose being defined, taking the respective advantages/disadvantages into account. Remarkable progress in the calculation of heavy ion reactions and transportation has been made during the last decade (see, for example, Sihver et al., 2007, 2008a, b, 2009; Sihver, 2008). In recent years, PHITS has received considerable attention due to its applications in heavy ion transport calculations. Several interaction models for calculating heavy ion reactions, such as JQMD and JAMQMD, are incorporated into PHITS. Consequently, it is possible to transport heavy ions with a considerably high reliability.

By taking advantage of the heavy ion transport models in PHITS, we have estimated neutron production by GCR protons and heavy components. Accurate calculation of the heavy ion reactions and transportation are important when estimating the neutron and gamma ray production from GCR. Although the amount of heavy nuclei $(Z \geq 2)$ in the GCR flux is small relative to that of protons, the neutron and gamma ray production rates by heavy ions are much larger than those by the protons. Therefore, neutron production from heavy ion reactions should be taken into account. The neutron production from alpha particles is of particular importance because alpha particles are more abundant than other heavy nuclei $(Z>2)$. The neutrons produced by the protons and alpha particles form the major part of the total neutron density profile produced by all the nuclides in the GCR. We have therefore focused on the production of neutrons from alpha particles in the GCR. To date, neutron production from alpha particles has been mainly estimated by scaling the calculation of protons (Dagge et al., 1991; Masarik and Reedy, 1996). The primary aim of our study was to compare, in detail, the estimation of neutron production from alpha particles by the proton scaling method with that by alpha particle interaction model.

We first calculated the neutron density as a function of depth (neutron density profile) produced by the interaction of GCR particles with lunar material. We then compared our calculated values with the experimental values obtained in the Apollo 17 Lunar Neutron Probe Experiment (LNPE) (Woolum et al., 1973, 1975; Woolum and Burnett, 1974a, b). Since neutron production affects gamma ray production, our calculation is a major contribution in furthering neutron and gamma ray spectroscopy and space dosimetry on the
Moon.

\section{Simulation Procedure \\ 2.1 PHITS}

The simulations described in this paper were performed with the three-dimensional Monte Carlo Particle and Heavy Ion Transport code System (PHITS), which was developed by Niita et al. (2006) and Iwase et al. (2002). PHITS ver. 2.13 was used throughout the work reported here. PHITS has several models for calculating nuclear reactions and the transportation of energetic particles, such as protons, neutrons, heavy ions, and some exotic particles $>10 \mathrm{MeV} / \mathrm{n}$ (heavy ions) and $>1 \mathrm{MeV}$ (otherwise) in matter. In addition, PHITS can deal with the complex motion of lowenergy neutrons $<20 \mathrm{MeV}$ in a similar manner as the MCNP4C code. PHITS has been successfully used for many radiation transport analyses of the space radiation environment both inside and outside a spacecraft (the reader is referred to Sato et al., 2006, 2008a; Sihver et al., 2009; Gustafsson et al., submitted; Sihver et al., submitted). Thus, we concluded it is possible to calculate the neutron production in the lunar subsurface accurately using the PHITS.

\subsection{Geometry and material}

The simulation procedure for neutron production in the lunar subsurface basically follows the conditions under which the LNPE was performed from Extra-Vehicular Activity (EVA) 1 to EVA 3 in the Apollo 17 mission in 1973.

The LNPE measured equilibrium neutrons within the first $400 \mathrm{~g} / \mathrm{cm}^{2}$ using two kinds of track detectors, namely, muscovite mica fission detectors with ${ }^{235} \mathrm{U}$ targets and cellulose triacetate (Triafol TN) plastic detectors with ${ }^{10} \mathrm{~B}$ targets (Woolum et al., 1973). A total of eight ${ }^{235} \mathrm{U}$ targets and 23 ${ }^{10} \mathrm{~B}$ targets were attached to a $2-\mathrm{m}$-long rod. The rod was then inserted into the drill hole created in the lunar subsurface. The neutron capture rates of ${ }^{235} \mathrm{U}$ and ${ }^{10} \mathrm{~B}$ targets were obtained from the track densities of fission fragments emitted from ${ }^{235} \mathrm{U}$ induced by neutrons with about $5 \mathrm{meV}$ to $5 \mathrm{keV}$ energy and the alpha and some Li recoils emitted via the ${ }^{10} \mathrm{~B}(n, \alpha) \mathrm{Li}$ reaction induced by neutrons with about $5 \mathrm{meV}$ to $500 \mathrm{eV}$ energy (Woolum et al., 1975). Finally, the ${ }^{235} \mathrm{U}$ fission rate and neutron density as a function of depth were measured by converting the ${ }^{235} \mathrm{U}$ and ${ }^{10} \mathrm{~B}$ neutron capture rates, respectively, using the neutron capture cross sections. The instrument and its measurement method are described in more detail in Woolum et al. (1973). Data analysis and the estimation of experimental error can be found in Woolum and Burnett (1974a) and Woolum et al. (1975). In this study, our calculation was compared with the neutron density profile obtained with Triafol TN detectors. The data labeled "Woolum" in this paper were taken from Woolum et al. (1975) and are the same as those used by McKinney et al. (2006). An overall experimental error of $15 \%$ estimated by Woolum and Burnett (1974b), which includes errors of track measurements (7-9\%) and several correction effects for conversion to neutron densities, was used in the data (Woolum et al., 1975).

The geometry of the target and beam source in our simulation is modeled after the relationship between the lunar body and the GCR. First of all, a sphere with a radius of 
Table 1. Abundances (wt \%) and densities of the lunar subsurface used in our calculations. These data were taken from the Lunar Neutron Probe Experiment borehole analysis by McKinney et al. (2006). * Read 4.174E-1 as $4.174 \times 10^{-1}$.

\begin{tabular}{ccccc}
\hline $\begin{array}{c}\text { Depth }(\mathrm{cm}) \\
\left(\text { Density }\left(\mathrm{g} / \mathrm{cm}^{3}\right)\right)\end{array}$ & $\begin{array}{c}0-22 \\
(1.76)\end{array}$ & $\begin{array}{c}22-71 \\
(2.11)\end{array}$ & $\begin{array}{c}71-224 \\
(1.78)\end{array}$ & $(1.79)$ \\
\hline Element & \multicolumn{2}{c}{ Abundance $(\mathrm{wt} \%)$} \\
\hline $\mathrm{O}$ & $4.174 \mathrm{E}-01^{*}$ & $4.156 \mathrm{E}-01$ & $4.230 \mathrm{E}-01$ & $4.264 \mathrm{E}-01$ \\
$\mathrm{Na}$ & $2.923 \mathrm{E}-03$ & $3.136 \mathrm{E}-03$ & $3.075 \mathrm{E}-03$ & $3.455 \mathrm{E}-03$ \\
$\mathrm{Mg}$ & $6.162 \mathrm{E}-02$ & $6.026 \mathrm{E}-02$ & $6.156 \mathrm{E}-02$ & $6.091 \mathrm{E}-02$ \\
$\mathrm{Al}$ & $6.061 \mathrm{E}-02$ & $5.977 \mathrm{E}-02$ & $7.384 \mathrm{E}-02$ & $7.550 \mathrm{E}-02$ \\
$\mathrm{Si}$ & $1.903 \mathrm{E}-01$ & $1.896 \mathrm{E}-01$ & $1.967 \mathrm{E}-01$ & $2.022 \mathrm{E}-01$ \\
$\mathrm{~K}$ & $7.262 \mathrm{E}-04$ & $7.896 \mathrm{E}-04$ & $9.201 \mathrm{E}-04$ & $1.632 \mathrm{E}-03$ \\
$\mathrm{Ca}$ & $7.541 \mathrm{E}-02$ & $7.668 \mathrm{E}-02$ & $8.020 \mathrm{E}-02$ & $7.707 \mathrm{E}-02$ \\
$\mathrm{Ti}$ & $5.144 \mathrm{E}-02$ & $4.905 \mathrm{E}-02$ & $3.380 \mathrm{E}-02$ & $3.198 \mathrm{E}-02$ \\
$\mathrm{Cr}$ & $2.872 \mathrm{E}-03$ & $3.090 \mathrm{E}-03$ & $2.641 \mathrm{E}-03$ & $2.545 \mathrm{E}-03$ \\
$\mathrm{Mn}$ & $1.764 \mathrm{E}-03$ & $1.778 \mathrm{E}-03$ & $1.519 \mathrm{E}-03$ & $1.458 \mathrm{E}-03$ \\
$\mathrm{Fe}$ & $1.350 \mathrm{E}-01$ & $1.403 \mathrm{E}-01$ & $1.228 \mathrm{E}-01$ & $1.169 \mathrm{E}-01$ \\
$\mathrm{Sm}$ & $8.334 \mathrm{E}-06$ & $7.747 \mathrm{E}-06$ & $7.343 \mathrm{E}-06$ & $1.051 \mathrm{E}-05$ \\
$\mathrm{Eu}$ & $1.816 \mathrm{E}-06$ & $1.782 \mathrm{E}-06$ & $1.545 \mathrm{E}-06$ & $1.700 \mathrm{E}-06$ \\
$\mathrm{Gd}$ & $1.100 \mathrm{E}-05$ & $1.058 \mathrm{E}-05$ & $9.818 \mathrm{E}-06$ & $1.355 \mathrm{E}-05$ \\
$\mathrm{Th}$ & $9.449 \mathrm{E}-07$ & $8.022 \mathrm{E}-07$ & $1.382 \mathrm{E}-06$ & $3.006 \mathrm{E}-06$ \\
\hline
\end{tabular}

Table 2. Parameters of proton and alpha particle energy spectra, and total fluxes (particle $/ \mathrm{cm}^{2} / \mathrm{s}$ ) used in our calculations. The total fluxes were obtained by integration of the spectra ranging from $10 \mathrm{MeV} / \mathrm{n}$ to $20 \mathrm{GeV} / \mathrm{n}$. ${ }^{*}$ Units are particle $/ \mathrm{cm}^{2} / \mathrm{s}$.

\begin{tabular}{ccccccccc}
\hline Particle & $\begin{array}{c}C \\
\left(1 / \mathrm{cm}^{2} / \mathrm{s} / \mathrm{MeV}\right)\end{array}$ & $\begin{array}{c}a \\
(\mathrm{MeV})\end{array}$ & $\begin{array}{c}b \\
(1 / \mathrm{MeV})\end{array}$ & & $\begin{array}{c}\text { Flux }^{*} \\
(\phi=500)\end{array}$ & $\begin{array}{c}\text { Flux }^{*} \\
(\phi=550)\end{array}$ & $\begin{array}{c}\text { Flux }^{*} \\
(\phi=600)\end{array}$ \\
\hline Proton & $1.24 \mathrm{E}+06$ & 780 & $2.50 \mathrm{E}-04$ & 2.65 & 3.16 & 2.88 & 2.63 \\
Alpha & $2.26 \mathrm{E}+05$ & 660 & $1.40 \mathrm{E}-04$ & 2.77 & 0.233 & 0.21 & 0.19 \\
\hline
\end{tabular}

$1738 \mathrm{~km}$, which is the same size as the moon, was designated the target. Then, the three concentric spheres were configured inside the lunar sphere to reproduce differences in material and the density dependent on the depth from the surface. The materials and densities in the lunar body used as input are summarized in Table 1. These data were obtained by McKinney et al. (2006) based on data obtained from the Soil Mechanics experiment in the Apollo 17 mission carried out by Carrier (1974) and Mitchell et al. (1974). The temperature of the material was set at $250 \mathrm{~K}$, as suggested by Woolum and Burnett (1974a). The actual temperature may vary under $333 \mathrm{~K}$, which the LNPE temperature indicators suggested as a maximum, because the LNPE was carried out for $49 \mathrm{~h}$. However, based on the results of our preliminary tests, differences in material temperature primarily affect the shape of the low-energy neutron spectrum but have small effects on the neutron density. Similar results have also been reported by other investigators (Woolum et al., 1975; Dagge et al., 1991; Masarik and Reedy, 1996). Therefore, exact information on the temperature is not important. As the beam source, a shell with a radius of $1738+$ $0.001 \mathrm{~km}$ was configured outside the lunar sphere. Protons and alpha particles were used as the source particles from the GCR. The energy spectra of the proton and alpha particles were generated with the analytical functions described in the following section. The particles are created within PHITS as an external isotropic source on the shell and then originate randomly and inwardly from the shell. The space between the lunar surface and the shell with the source particles was set as a vacuum.

\subsection{Beam source}

A form of analytical functions for the energy spectra of proton and alpha particles in GCR has been widely accepted as the means to vary the parameters (Castagnoli and Lal, 1980; Dagge et al., 1991; Masarik and Reedy, 1996; McKinney et al., 2006). The $4 \pi$ GCR flux $J$ (particle/(MeV/n) $/ \mathrm{cm}^{2} / \mathrm{s}$ ) is expressed by

$$
J(E, \phi)=C \frac{E\left(E+2 m_{\mathrm{p}} c^{2}\right)(E+\chi+\phi)^{-\gamma}}{(E+\phi)\left(E+2 m_{\mathrm{p}} c^{2}+\phi\right)},
$$

where $C\left(1 / \mathrm{cm}^{2} / \mathrm{s} / \mathrm{MeV}\right)$ is a normalization factor, $E$ $(\mathrm{MeV} / \mathrm{n})$ is the kinetic energy of GCR nuclei, $\phi(\mathrm{MV})$ is the solar modulation parameter to characterize the effect of solar activity, $m_{\mathrm{p}}\left(\mathrm{MeV} / \mathrm{c}^{2}\right)$ is the mass of the proton, $c$ is the velocity of light, $\chi=a \exp (-b E)$, and the remaining parameters, such as $a, b$, and $\gamma$, are provided in Table 2 . These parameters have been described in detail for protons (Castagnoli and Lal, 1980) and alpha particles (Lal, 1985). When the LNPE was carried out, $\phi$ corresponds to $550 \pm 50$ MV. The determination of $\phi$ was studied by McKinney et al. (2006) in detail. Therefore, we used the energy spectra for proton and alpha particle at $\phi=550 \pm 50 \mathrm{MV}$. As can be seen in Table 2, the variability in total flux at $\phi=500$ and $600 \mathrm{MV}$ ranges over $10 \%$ from 1 at $\phi=550 \mathrm{MV}$, respectively, because GCR particles with energies of $<10 \mathrm{GeV} / \mathrm{n}$ are extremely affected by the solar activity. Therefore, neutron density also depends on the value of $\phi$. In our calculation, the energy cut-off of the generated source particles was set at a minimum of $10 \mathrm{MeV} / \mathrm{n}$ and a maximum of 
Table 3. Interaction model options in the PHITS (* denotes the default model).

\begin{tabular}{ll}
\hline Particle & Model options $(E$ denotes the kinetic energy of particle $)$ \\
\hline Proton & Bertini Free $(1 \mathrm{MeV}<E<3.5 \mathrm{GeV})+{ }^{*} \mathrm{JAM}(E>3.5 \mathrm{GeV})$ \\
& ${ }^{*}$ Bertini Cugnon Old $(1 \mathrm{MeV}<E<3.5 \mathrm{GeV})+{ }^{*} \mathrm{JAM}(E>3.5 \mathrm{GeV})$ \\
& Bertini Cugnon New $(1 \mathrm{MeV}<E<3.5 \mathrm{GeV})+{ }^{*} \mathrm{JAM}(E>3.5 \mathrm{GeV})$ \\
& Isober $(1 \mathrm{MeV}<E<1 \mathrm{GeV})+{ }^{*}$ Bertini Cugnon Old $(1 \mathrm{GeV}<E<3.5 \mathrm{GeV})+{ }^{*} \mathrm{JAM}(E>3.5 \mathrm{GeV})$ \\
& JAM $(E>1 \mathrm{MeV})$ \\
& JQMD $(E>1 \mathrm{MeV})$ \\
& JAMQMD $(E>1 \mathrm{MeV})$ \\
& JQMD $(E>10 \mathrm{MeV} / \mathrm{n})$ \\
Alpha & JAMQMD $(E>10 \mathrm{MeV} / \mathrm{n})$ \\
\hline
\end{tabular}

\section{$20 \mathrm{GeV} / \mathrm{n}$}

The beam was projected inwardly from the shell with the angular probability distribution of the cosine, i.e., $p(\cos \theta)=\cos \theta$. Here, $\theta$ is the angle between the particle incident direction and normal to the shell surface. The cosine angular distribution generated from the shell makes the density of the particles within the sphere uniform. As such, it has been ensured that the lunar surface is bombarded with the GCR nuclei isotropically.

In the simulations described in this paper, typically 100,000 and 25,000 source particles were used for proton and alpha particles, respectively, and the statistical error was estimated to be $<1 \%$.

\subsection{Nuclear interaction model}

The different theoretical models used for the protoninduced and alpha-induced nuclear interactions in our study are summarized in Table 3. Below $3.5 \mathrm{GeV}$, PHITS uses the Bertini intranuclear cascade model as a default option for the nucleon-induced interaction. There are three different optional parameterizations of the nucleon-nucleon interaction cross section data used in the Bertini model, namely, "Cugnon Free", "Cugnon Old", and "Cugnon New", respectively. PHITS uses the Cugnon Old parameterization step in the Bertini model as the default. Although the Bertini model is a default model as far as these cross-section data exist $(1 \mathrm{MeV}<E<3.5 \mathrm{GeV}$ ), it is no longer applicable $>3.5 \mathrm{GeV}$, when the default model switches to the JAM (Jet AA Microscopic Transport Model), which is an original hadronic cascade model (Nara et al., 2000; Niita et al., 2001, 2006). However, it is possible to select other models, such as JAM, JQMD (JAERI Quantum Molecular Dynamics), and JAMQMD for the full energy range $(1 \mathrm{MeV}<E<200 \mathrm{GeV}$ ) (Niita et al., 1995). For the nucleus-induced interaction, PHITS uses JQMD $<3.5 \mathrm{GeV} / \mathrm{n}$ and JAMQMD $>3.5 \mathrm{GeV} / \mathrm{n}$ as a default. Similarly to the proton interaction model, it is possible to use JQMD and JAMQMD for the full energy range (10 MeV/n $<E<100 \mathrm{GeV} / \mathrm{n}$ ). JQMD describes the behavior of the nucleon-nucleus and nucleus-nucleus reactions by solving the equation of motion for each nucleon-nucleon interaction. JAMQMD is basically the same as JQMD, with the exception that the nucleon-nucleon interaction cross section is calculated using the JAM model. In this work, the calculations for both protons and alpha particles were verified as shown below.

\subsection{Simulated geometry}

In the target sphere, 55 concentric spheres were configured in order to calculate the neutron fluxes at various depths. These spheres were set to depths of $1-10 \mathrm{~cm}$, and then the neutron flux crossing the spherical surface was calculated at each depth. In this way, neutron fluxes were obtained at 55 points from the surface down to the first $3 \mathrm{~m}$, corresponding to about $500 \mathrm{~g} / \mathrm{cm}^{2}$ in the lunar subsurface. The neutron density $\left(\rho_{\text {neutron }}\right)$ at a given depth $(d)$ was obtained by conversion from the neutron flux $\left(F_{\text {neutron }}\right)$ at $d$ with energies $(E)<500 \mathrm{eV}$ using the neutron velocity $(v)$. This conversion is given by

$$
\rho_{\text {neutron }}(d)=\int_{0}^{500 \mathrm{eV}} \frac{F_{\text {neutron }}(d, E)}{v(E)} d E .
$$

\section{Neutron Density Profile Estimated by GCR Protons Using a Scale Factor}

The first step was to calculate the neutron density profile using the conventional estimation method, which is scaling of neutron production from the GCR protons (Dagge et al., 1991; Masarik and Reedy, 1996). Since many transportation codes can not transport particles heavier than protons, a scale factor to estimate the neutron contribution from the alpha particles has been used for many years. This scale factor corresponds to the average ratio of alpha particle to the proton in terms of neutron production efficiency in the full energy range of GCR spectra. When we define the scale factor as $x$ at $\phi=550 \mathrm{MV}$, the neutron production from protons and alpha particles will increase by a factor of $(2.88+0.210 \times x) / 2.88$ compared to that from only protons (note 2.88 and 0.210 are the total fluxes of protons and alpha particles (particle $/ \mathrm{cm}^{2} / \mathrm{s}$ ), respectively). For example, Reedy and Arnold (1972) estimated the scale factor to be 2.8 for energies $>1 \mathrm{GeV} / \mathrm{n}$, Masarik and Reedy (1996) invoked the scale factor of 3.0 (Reedy, 2009) using the LCS (Prael and Lichtenstein, 1989), and Dagge et al. (1991) reported 3.5 to be the optimum factor using the HERMES code (Prael and Lichtenstein, 1989; Prael, 1993), which supports the alpha particle irradiation. By indirect means, Yamashita et al. (2006) measured the factor to be 3.5 from the ratio of the efficiencies of the alpha particle and proton to produce gamma rays. On the other hand, McKinney et al. (2006) suggested a factor of 3.8 using a preliminary version of MCNPX 2.6.0, which supports the alpha particle transportation. In this section, we calculated the neutron 


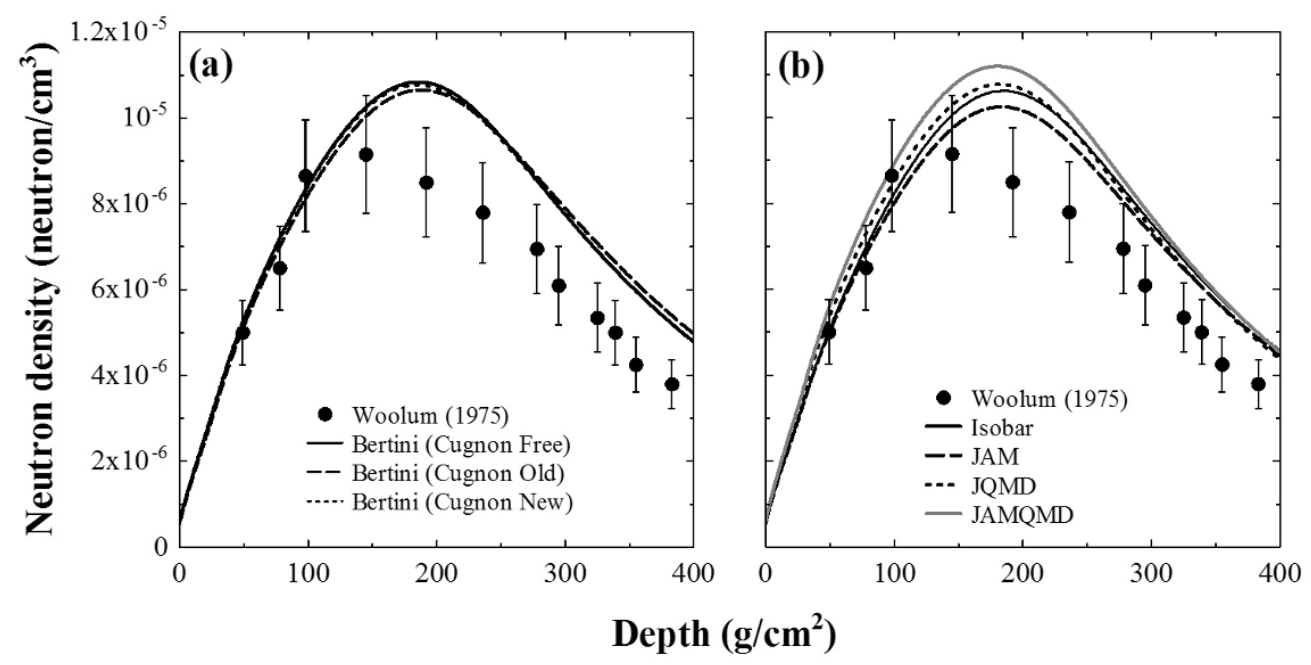

Fig. 1. Comparison of calculated neutron density profiles with the experimental results reported by Woolum et al. (1975). The calculations were performed using seven different kinds of proton interaction models; (a) Cugnon Free, Cugnon Old, Cugnon New, (b) Isobar, JAM, JQMD, JAMQMD. In both figures, neutron production from alpha particles in the GCR was estimated using a scale factor of 3.5; this factor was based on that for protons in the GCR.

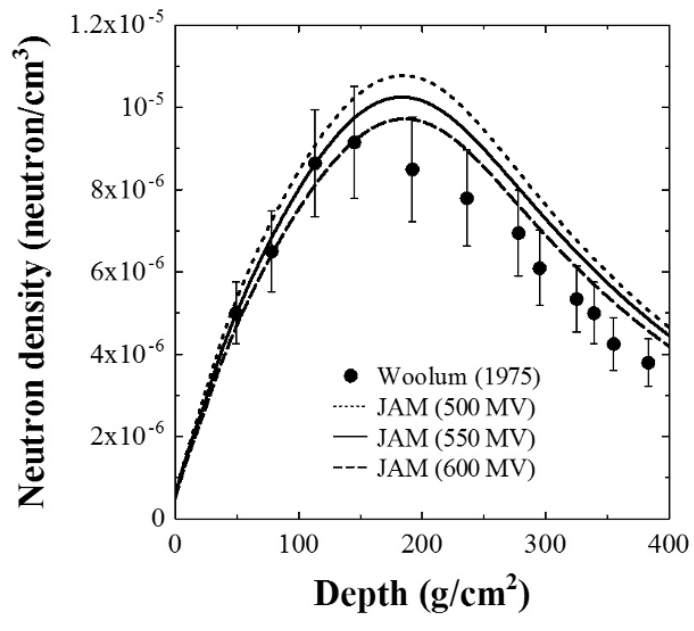

Fig. 2. Comparison of calculated neutron density profiles with the experimental results resported by Woolum et al. (1975). The calculations were performed using the JAM interaction model for the three different solar modulation parameters. Neutron production from alpha particles in the GCR was estimated using a scale factor of 3.5; this factor was based on that for protons in the GCR.

production from proton and alpha particles in GCR using the scale factor of 3.5 .

The calculated neutron density profiles are shown in Figs. 1 and 2. The results calculated by all patterns of models in Table 3 at $\phi=550 \mathrm{MV}$ are shown in Fig. 1(a) and (b). The trend that peaks of neutron density profiles exist around $150-180 \mathrm{~g} / \mathrm{cm}^{2}$ is similar to the experimental results. However, the greatest difference in the absolute values is that of $30 \%$ by JAMQMD around $200 \mathrm{~g} / \mathrm{cm}^{2}$ and $50 \%$ by Bertini Cugnon Old around $350 \mathrm{~g} / \mathrm{cm}^{2}$. Compared to the LNPE data, it is evident that JAM provides the best agreement. As mentioned above, the solar modulation parameter $\phi$ also has a large influence on neutron production. Therefore, we calculated the neutron density profile at $\phi=500,550$, and

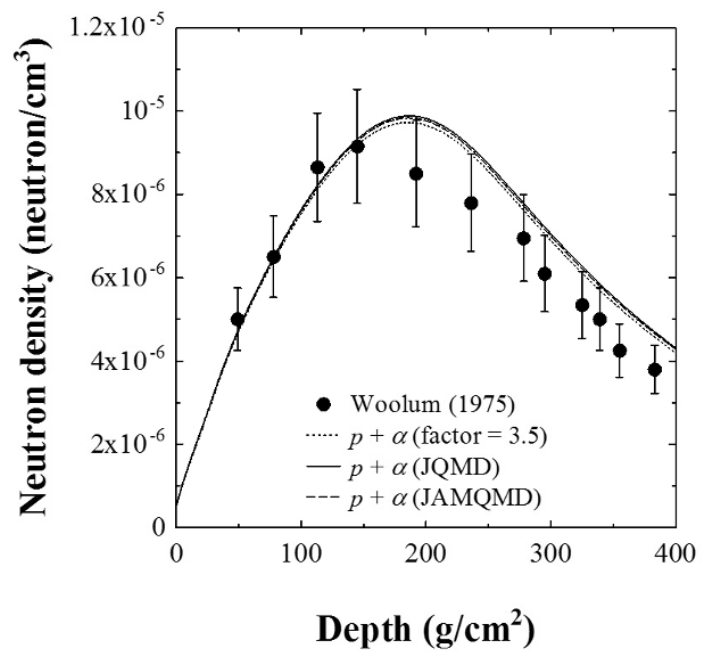

Fig. 3. Comparison of calculated neutron density profiles with the experimental results reported by Woolum et al. (1975). The calculations were performed using three modes at $\phi=600 \mathrm{MV}$. In the calculation using the scale factor, the neutron production from alpha particles in the GCR was estimated using a scale factor of 3.5; this factor was based on that for protons in the GCR.

$600 \mathrm{MV}$ using the JAM in Fig. 2. The agreement between the calculation at $\phi=600 \mathrm{MV}$ and experimental values is much improved. Thus, our calculation results successfully reproduced the LNPE data within the measurement error of $15 \%$.

\section{Neutron Production from the GCR Alpha Par- ticles}

In this section, we report our estimation of neutron production from the GCR alpha particles using two different heavy ion interaction models: JQMD and JAMQMD (see Table 3). The calculated results of neutron density 


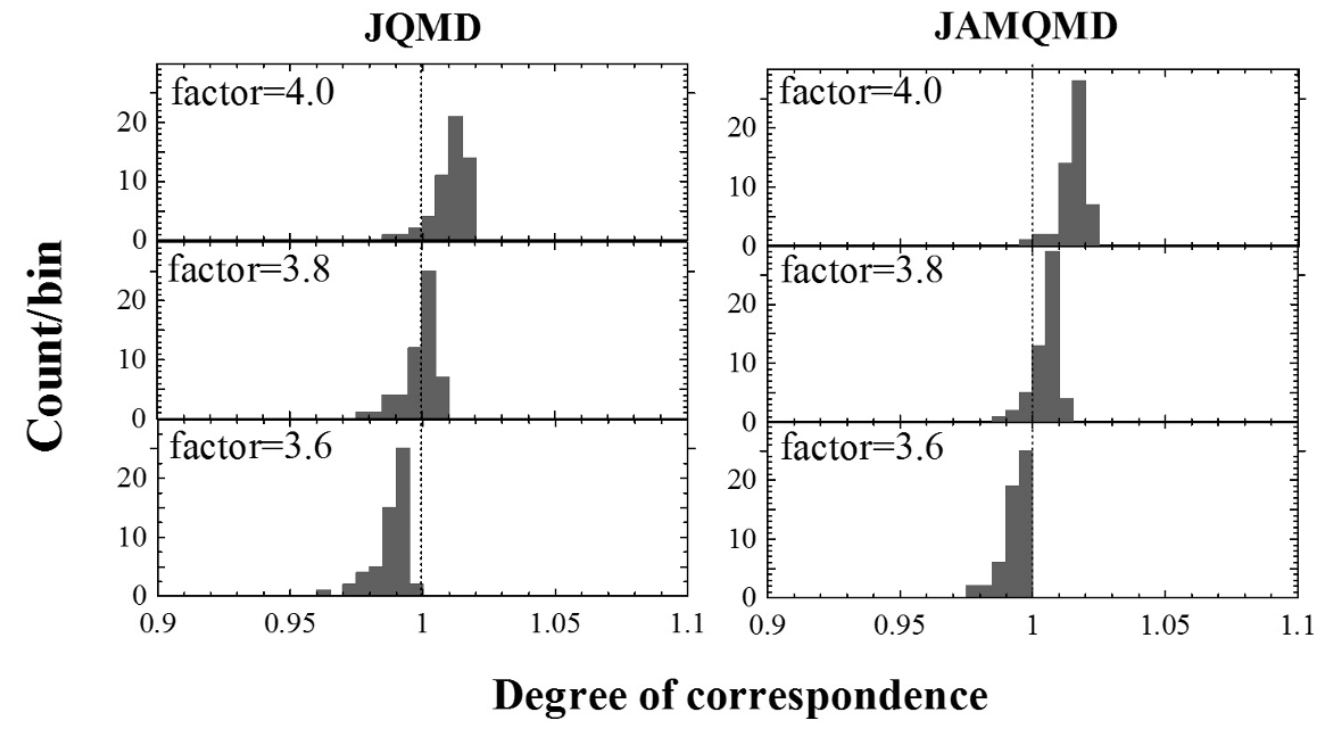

Fig. 4. Degree of correspondence of neutron density profiles calculated at 55 points from the lunar surface to the first $3 \mathrm{~m}$ at $\phi=600 \mathrm{MV}$ by the proton scaling method with those calculated by the two different alpha particle interaction models of JQMD and JAMQMD. The degree of correspondence was defined as the ratio of the result obtained by the alpha particle interaction model to the proton scaling method.
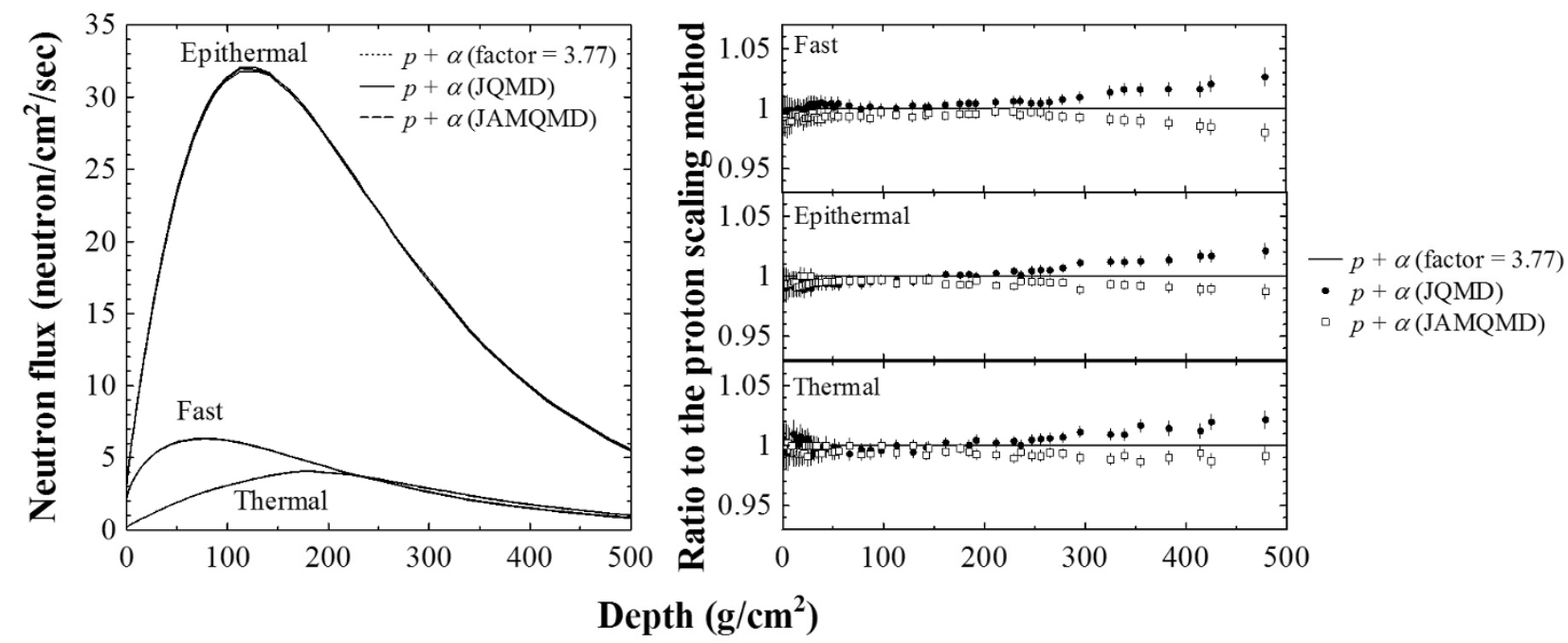

Fig. 5. (a) Distributions of neutron fluxes in the lunar subsurface calculated by the proton scaling method and alpha particle interaction models. Note that all of the patterns of calculations overlap in the individual distributions of fast, epithermal, and thermal neutrons, respectively. (b) Comparisons of neutron fluxes calculated at 55 points from the lunar surface to the first $3 \mathrm{~m}$ by the alpha particle interaction models of JQMD and JAMQMD with that by the proton scaling method.

profile using JQMD and JAMQMD for alpha particles are shown in Fig. 3. In these calculations, JAM was used for the proton interaction. The results by JQMD and JAMQMD showed a good agreement with each other, although the results from both JQMD and JAMQMD are a few percentage points higher than that achieved by the proton scaling method. This difference suggests that a scale factor of 3.5 is a little low to estimate correctly the contribution from the GCR alpha particles. To study the contribution of alpha particles in more detail, we changed the scale factor to 3.6, 3.8, and 4.0, respectively. Comparisons of the neutron density profile by the proton scaling method with those using JQMD and JAMQMD are shown in Fig. 4. As can be seen in Fig. 4, a scale factor of 3.8 provides a better agree- ment with the calculations performed with both the JQMD and JAMQMD. The standard deviations of such distributions are about $1 \%$, suggesting that no special difference was found between the proton scaling method and the alpha particle interaction models. We conclude that 3.81 and 3.72 are the best factors by which to obtain the best fits to the results by JQMD and JAMQMD, respectively, in the more detailed comparison. More detailed differences from the view of thermal $(E \leq 1 \mathrm{eV})$, epithermal $(1 \mathrm{eV}<E \leq$ $1 \mathrm{MeV})$, and fast neutrons $(E>1 \mathrm{MeV})$ using the scale factor of 3.77 are shown in Fig. 5(a) (3.77 is the mean of 3.81 and 3.72). Detailed comparisons are made in Fig. 5(b). In this figure, one can see that there is an agreement between the proton scaling method and the alpha interaction models 


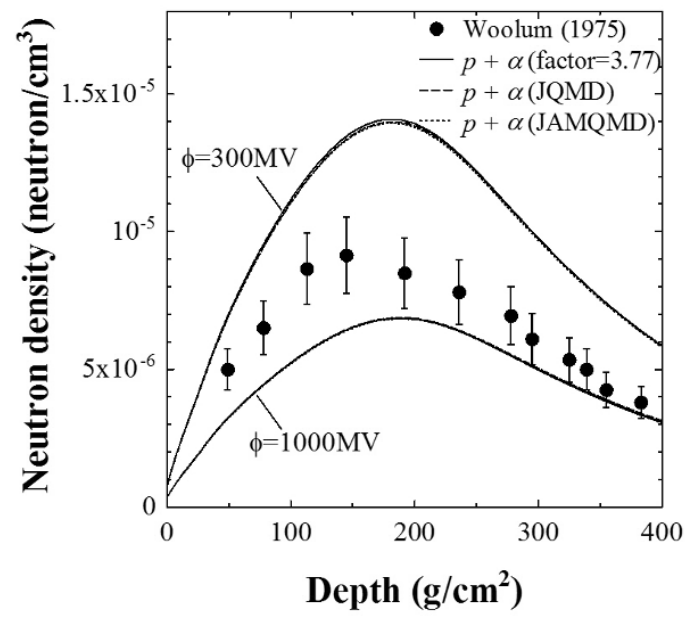

Fig. 6. Comparisons of calculated neutron density profiles by the proton scaling method and the alpha particle interaction models (JQMD and JAMQMD) in the periods of minimum and maximum activities of the solar cycle.

within an error of $1 \%<250 \mathrm{~g} / \mathrm{cm}^{2}$ for all energy ranges. On the other hand, the discrepancies increase up to a few percent points $>250 \mathrm{~g} / \mathrm{cm}^{2}$. However, these discrepancies result in only a small specific difference in the actual application method, e.g., neutron and gamma ray spectroscopy and space dosimetry.

\section{Discussion}

\subsection{Correlation between the scale factor and solar cy-} cle

We have determined the scale factor for estimating the neutron production from alpha particles in the lunar subsurface at $\phi=600 \mathrm{MV}$, or around a period of average activity of the solar cycle. In this subsection, the validity of the scale factor is verified at the solar minimum $(\phi=300 \mathrm{MV})$ and solar maximum $(\phi=1000 \mathrm{MV})$ of solar cycle activity. The calculated results at $\phi=300$ and $1000 \mathrm{MV}$ are shown in Fig. 6. We found that a scale factor of 3.77 reproduced neutron production from alpha particles for the cases of both $\phi=300$ and $1000 \mathrm{MV}$ within an accuracy of $1 \%$ (standard deviation). Therefore, the scale factor can be applied throughout the entire period of solar cycle. When we use our estimated scale factor of 3.77, neutron production from the GCR particle (proton + alpha particles) increases by a factor of $1.29,1.27$, and 1.26 for $\phi=300,600$, and $1000 \mathrm{MV}$, respectively, compared to the one from only protons.

\subsection{Reliability of transport models}

As shown in Fig. 3, our calculation successfully reproduced the LNPE neutron density profile data within the measurement error of $15 \%$. However, there is still room for improvement of both the simulations and the evaluation of the measurements. For example, Woolum et al. (1975) suggested that the LNPE data may have 10-30\% systematic errors that result from the different observer-dependent criteria for nuclear track recognition. We therefore discuss possible options for improvement in this and the next subsection. We also discuss the reliability of our calculation from the points of uncertainty of the transport model and the GCR spectra.

In our calculations, the neutron density profile is directly dependent on the correlation of the neutron production cross section and the energy of the GCR particles. Therefore, we calculated the excitation functions of the total neutron production cross sections for protons and alpha particles on the lunar material (aluminum as an example). A thin cubic target made of aluminum, $4 \times 4 \times 4 \mu \mathrm{m}^{3}$, was established, and the source particles of protons and alpha particles were then generated at the point of $1 \mathrm{~cm}$ from the target. The space surrounding the target was a vacuum, and the target was bombarded with pencil and monochromatic beams that were perpendicular to the target surface. Thus, the total neutron production cross section for the incident particles on the Al target was determined from the numbers of source particles and from the neutrons produced. The geometry of this simulation can be found in more detail in Mancusi et al. (2007). The cross sections were calculated from $10 \mathrm{MeV} / \mathrm{n}$ to $10 \mathrm{GeV} / \mathrm{n}$.

The excitation functions of the total neutron production cross sections produced from protons and alpha particles reacting with aluminum targets are shown in Fig. 7(a) and (b), respectively. Appreciably differences can be observed for the production of both protons and alpha particles. However, the difference in the neutron density profiles between the alpha particle interaction models of JQMD and JAMQMD is extremely small, as shown in the Fig. 3, suggesting that the accuracy of those heavy ion reaction models does not greatly influence neutron production. On the other hand, as can be seen in Fig. 1, the differences in the calculated neutron density profiles between the proton interaction models of Cugnon Free, Cugnon Old, Cugnon New, Isobar, JAM, JQMD, and JAMQMD fluctuated within $20 \%$, which implies that these fluctuations are caused by differences in the calculated energy-dependent cross sections in Fig. 7(a). Consequently, it is important to use an accurate proton interaction model - and not the alpha particle interaction model - to be able to calculate the cross sections at all energy ranges. When the proton interaction models that differ from JAM are used, the calculated results do not agree with the LNPE data. However, all of the calculation patterns agree with the LNPE data within $30 \%$ systematic error. Benchmarking and improvement of these models are therefore important. At the present time, we do not have any means to determine the best model for our calculation. In the near future, therefore, our aim must be to carefully benchmark the different models available in PHITS against the measurement of neutron production double differential cross sections for nuclear spallation reactions induced by $0.8,1.5$ and $3.0 \mathrm{GeV}$ protons, respectively (Ishibashi et al., 1997). In addition, the Japanese Evaluated Nuclear Data Library High Energy File (JENDL-HE) has recently become available (Fukahori et al., 2002; Watanabe et al., 2005). It has been frequently used to reproduce various experimental values (Sato and Niita, 2006; Sato et al., 2008b). We are in progress of incorporating the JENDL-HE into our calculation. These results will be reported in future publications. 

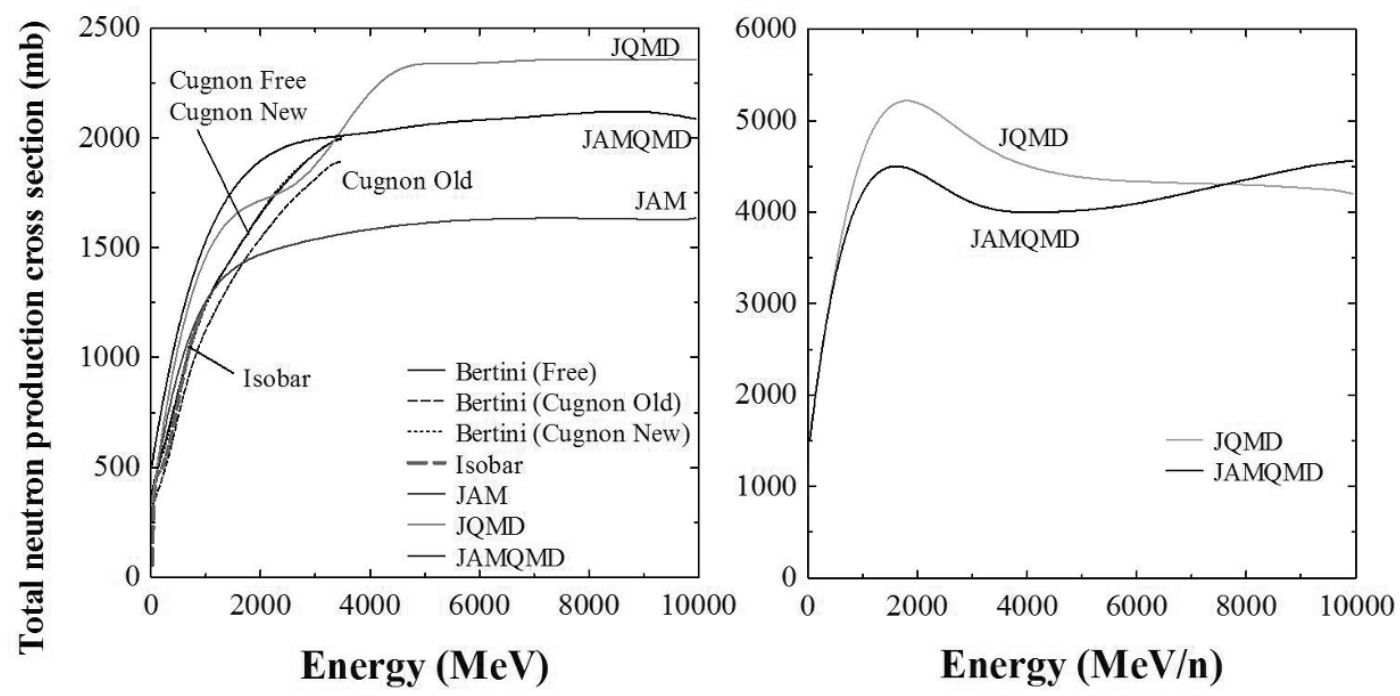

Fig. 7. (a) Excitation functions of the total neutron production cross section for protons on the Al target calculated using seven different interaction models. (b) Excitation functions of the total neutron production cross section for alpha particles on the Al target using JQMD and JAMQMD.

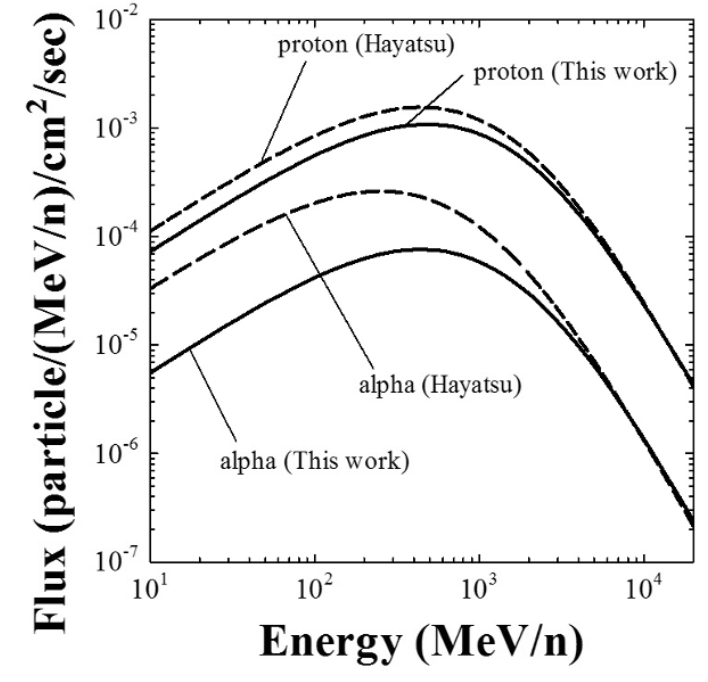

Fig. 8. Comparison of the GCR proton and alpha particle spectra used in this work and given by Hayatsu et al. (2008).

\subsection{Reliability of the GCR spectra}

We emphasize the importance of accurate GCR spectral data for the precise calculation of the neutron production in the lunar subsurface. Since Simpson's first report on the GCR spectra (Simpson, 1983), many researchers have made calculations based on his spectra. However, some recent measurements of GCR spectra performed within the framework of the AMS, CAPRISE98, and BESS balloon experiments reveal a number of important discrepancies from Simpson's data (Alcaraz et al., 2000; Boezio et al., 2003; Shikaze et al., 2007; Hayatsu et al., 2008). For example, according to Hayatsu et al. (2008), the total fluxes of protons and alpha particles calculated from the GCR spectra $(10 \mathrm{MeV} / \mathrm{n}-20 \mathrm{GeV} / \mathrm{n})$ by BESS at $\phi=600 \mathrm{MV}$ are 3.37 and $0.346 \mathrm{particle} / \mathrm{cm}^{2} / \mathrm{s}$, which are 28 and $82 \%$, respectively, higher than the fluxes used in this paper (see Table 2).
Figure 8 shows the GCR proton and alpha particle spectra calculated in our work and by Hayatsu et al. (2008) The neutron density profiles using the GCR spectra given by Hayatsu et al. (2008) are shown in Fig. 9(a). These are apparently higher than the results from our primary calculations. However, we should note that the results using the GCR spectra by Hayatsu et al. also give good agreement with the LNPE experimental values, within the systematic error of 30\%, as shown in Fig. 9(b). Thus, the uncertainties in the GCR spectra may cause overestimations or underestimations of neutron production. Therefore, further studies of GCR spectra are needed for a precise estimation of radiation environment on the Moon.

Another interesting topic is how the difference in the scale factor is dependent on the GCR spectra. In Fig. 9(a), the estimation using the scale factor of 3.77 is described. However, this scale factor does not seem to reproduce neutron production from alpha particles. The best fit to the calculations by the alpha particle interaction models is achieved with a scale factor of 2.94. This is in agreement with a scale factor of 3.0 that was previously estimated by Masarik and Reedy (1996) and Reedy (2009). Thus, we can safely state that variations in the scale factor are largely dependent on the GCR spectra, thereby demonstrating the need for more accurate data on the GCR spectra. On the other hand, it is not necessary to use a scale factor when using a heavy ion interaction model. Therefore, the use of the heavy ion interaction model in the radiation environment on the Moon will give more accurate results when calculating the neutron production in the lunar subsurface.

\subsection{Applications and future prospects}

The suitability of PHITS for the simulation of neutron spectroscopy was verified by the calculations reported here. In this subsection, we discuss future prospects for additional calculations in the application fields of lunar science and space dosimetry using the PHITS.

For neutron spectroscopy, the leakage neutron spectrum from the lunar surface is essential. Therefore, we calcu- 


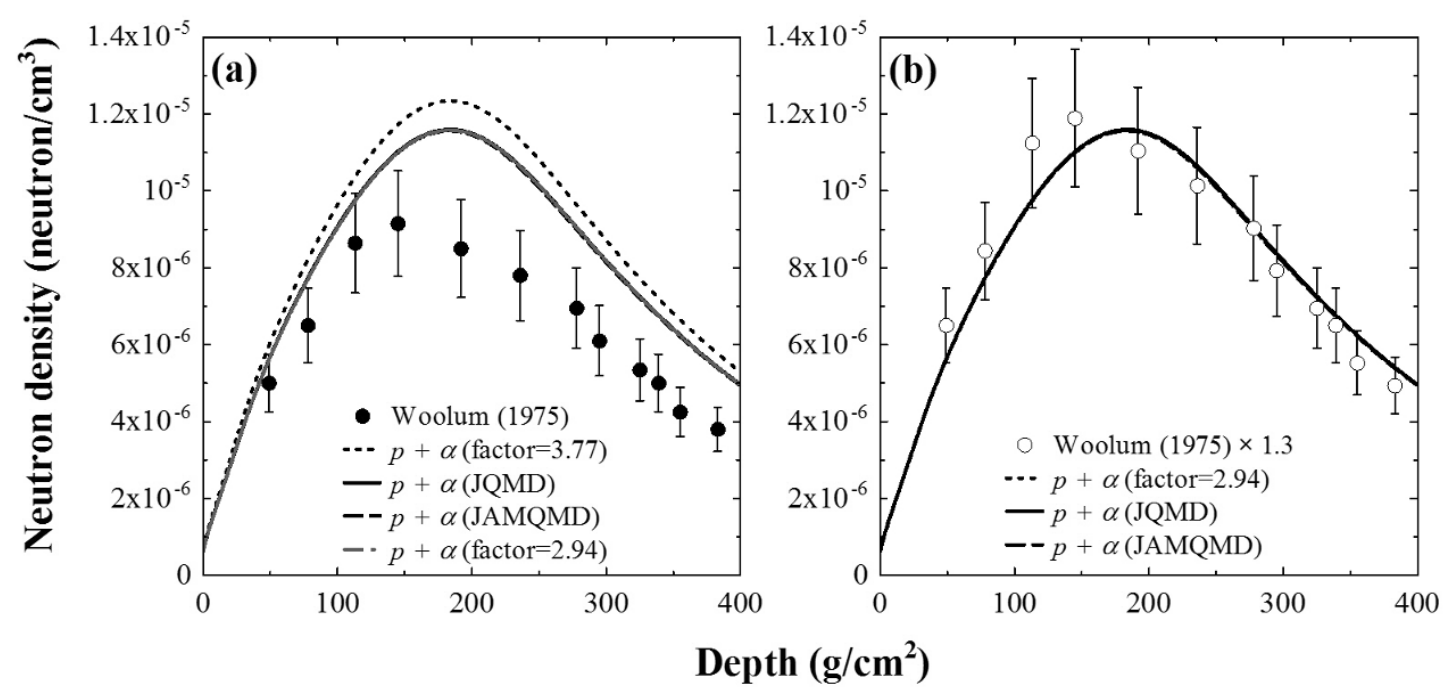

Fig. 9. (a) Neutron density profiles in the case of the GCR spectra reported by the BESS balloon experiment. (b) Comparison of neutron density profiles calculated using the GCR spectra by BESS with the experimental data reported by Woolum et al. (1975). The experimental data are multiplied by a factor of 1.3 to take into account the systematic error of $30 \%$.

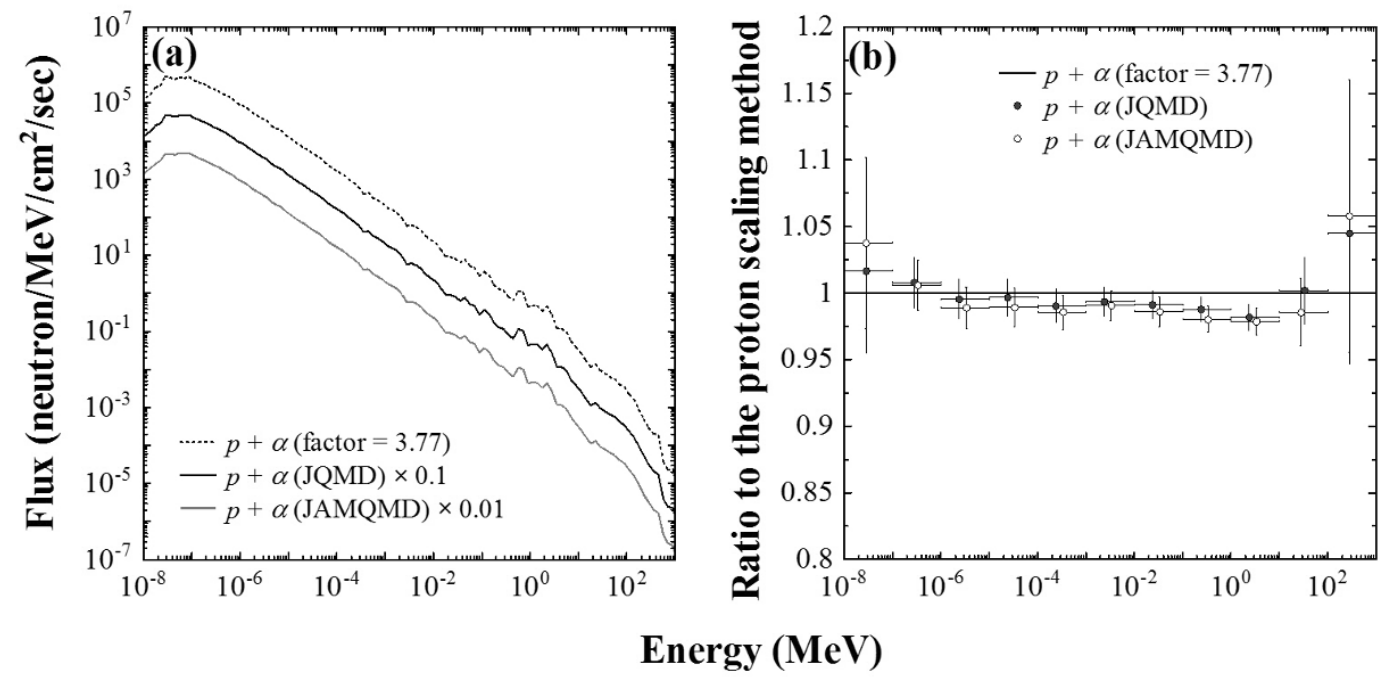

Fig. 10. (a) Leakage neutron spectra from the lunar surface calculated by the proton scaling method and the alpha particle interaction models (JQMD and JAMQMD). Note that the results by JQMD and JAMQMD are multiplied by a factor of 0.1 and 0.01 , respectively. (b) Comparison of the leakage neutron spectra in various energy ranges by the alpha particle interaction models with that by the proton scaling method. Longitudinal error bars are the statistical error.

lated the leakage neutron spectra from the lunar surface at $\phi=600 \mathrm{MV}$ by the proton scaling method and by the alpha particle interaction models in Fig. 10(a). As can be seen, there is a good agreement between all patterns of calculation results. Detailed comparisons are shown in Fig. 10(b), and the total neutron fluxes from the surface are shown in Table 4. It is obvious that no specific differences exist between the proton scaling method and the alpha particle interaction models.

Also, the consideration that the contribution of GCR nuclei is heavier than that of the alpha particles may be important. While GCR nuclei comprise only about $1 \%$ of GCR particles, they contain more than $10 \%$ of the nucleons in the GCR (Shapiro and Silberberg, 1970). However, it is more difficult to obtain accurate spectra of these particles
Table 4. Total leakage fluxes using different calculation modes. Percentages in parentheses indicate deviations from the proton scaling method.

\begin{tabular}{lc}
\hline \multicolumn{1}{c}{ Calculation mode } & Total leakage flux (neutrons $\left./ \mathrm{cm}^{2} / \mathrm{s}\right)$ \\
\hline Proton + Alpha (factor $=3.77)$ & 6.21 \\
Proton + Alpha (JQMD) & $6.17(-0.6 \%)$ \\
Proton + Alpha (JAMQMD) & $6.12(-1.5 \%)$ \\
\hline
\end{tabular}

than it is to obtain accurate spectra for the protons and alpha particles. Therefore, further detailed observations of GCR heavy components $(Z>2)$ over a wide energy range are needed. According to our preliminary calculations, if we assume (1) the same parameters and shape of the spectrum as those for the alpha particles used in this work and 
(2) total fluxes as those given by Simpson (1983), the neutron production in the lunar subsurface by those particles is $2 \%$ of that by protons and alpha particles. We can therefore conclude that neutron production from the GCR heavy components $(Z>2)$ is negligibly small.

Heavy ion interaction plays an essential role in terms of space dosimetry. According to Hayatsu et al. (2008), who considered only GCR as incident particles, secondary neutron and GCR heavy components $(Z \geq 2)$ contribute about 9 and $84 \%$, respectively, to the ambient dose equivalent on the lunar surface. Therefore, it is of importance that the transport and interactions of heavy ions are preformed with accuracy.

Finally, taking into account the availabilities in these applications fields, we conclude that PHITS is a suitable transport code to estimate the radiation environment, including the neutron components, in the lunar environment.

\section{Summary}

Galactic cosmic rays and solar energetic particles, which are main contributors to the neutron production in the lunar subsurface, include not only protons but also heavy components, such as alpha particles. It is important to be able to precisely estimate the neutron production from such components for the lunar spectroscopy and space dosimetry. Therefore, we calculated neutron production from the interactions of GCR protons and alpha particles with the lunar subsurface using the Particle and Heavy Ion Transport code System.

Neutron density profiles calculated by PHITS successfully reproduced the experimental data from the Apollo 17 Lunar Neutron Probe Experiment. In future studies, further detailed simulations of the emission and transport of radiation produced by cosmic radiation, including secondary products such as neutrons and gamma rays, are essential to determine the relationship between the chemical abundances and the intensities of gamma ray lines for planetary nuclear spectroscopy. This can also be applied to space dosimetry for the design of shielding and protection for a lunar base.

In this work, we found that the best scale factor to estimate neutron production from GCR alpha particles impinging on the lunar surface was $3.77 \pm 0.05$ using the conventional GCR spectra given by Castagnoli and Lal (1980) and Lal (1985). No major difference was found between neutron production from alpha particles calculated using the proton scaling method and that calculated using the alpha particle interaction models. The main reason for this lack of difference is that the flux of alpha particles is relatively small so the difference in neutron production by alpha particles from that by protons is not a large contributory factor. However, the factor cannot be determined in advance, as it depends on the GCR spectra. Actually, when using the GCR spectra based on the latest observation data by BESS, the best scale factor was 2.94. This is almost in agreement with the estimation by Masarik and Reedy (1996) and Reedy (2009). Thus, the variation in the scale factor is largely dependent on the GCR spectra. This clearly shows the need for more accurate data on the GCR spectra. On the other hand, it is not necessary to use a scale factor when using a heavy ion interaction model. Therefore, we conclude that the use of heavy ion interaction models, such as JQMD and JAMQMD, are important for estimating neutron production from the interactions of GCR with the lunar subsurface.

For future work in this area to be more accurate, two main uncertainties should be decreased. The uncertainties in the GCR spectra need to be decreased because these may cause overestimations or underestimations in the calculations. Improved modeling of GCR spectra has been recently reported by some investigators (Alcaraz et al., 2000; Boezio et al., 2003; Shikaze et al., 2007). Further detailed study on GCR spectra is necessary. On the other hand, it is also necessary to evaluate the accuracy of the different proton interaction models available in PHITS for neutron production. In the near future, we therefore aim to carefully benchmark the different models available in PHITS against the measurement of neutron-production double-differential cross sections for nuclear spallation reactions induced by $0.8,1.5$, and $3.0 \mathrm{GeV}$ protons (Ishibashi et al., 1997). We will also compare the calculated results using the Japanese Evaluated Nuclear Data Library High Energy File (JENDL$\mathrm{HE}$ ). Those results will be reported in a future publication.

Acknowledgments. We would like to express our thanks to the developers of the PHITS code for their kind support in its use. We also would like to thank Dr. S. Kunieda (JAEA, Japan) and Mr. H. Kitamura (NIRS, Japan) for their valuable discussions on the JENDL High Energy Nuclear Data Library and the design of the geometries used in this simulation, respectively. Moreover, we would like to express our thanks to Dr. R. C. Reedy (Planetary Science Institute, USA) and Dr. T. Sato (JAEA, Japan) for their very helpful comments. S. Ota is supported by the Practical Training Program for Doctoral Students at Waseda University. S. Kobayashi is supported by Research Fellowships of the Japan Society for the Promotion of Science (JSPS) for Young Scientist. L. Sihver would also like to acknowledge, with much gratitude, the support from JSPS, which made it possible for him to spend two very fruitful and interesting months as a short-term JSPS Fellow at NIRS, in 2009. This paper is a part of the outcome of research performed under a Waseda University Grant for Special Research Projects (Project number: 2009A-956).

\section{References}

Adams, J. H., M. Bhattacharya, Z. W. Lin, G. Pendleton, and J. W. Watts, The ionizing radiation environment on the moon, Adv. Space Res., 40, 238-341, 2007.

Agostinelli, S. et al., Geant4-a simulation toolkit, Nucl. Instr. Meth. A, 506, 250-303, 2003.

Alcaraz, J. et al., Cosmic protons, Phys. Lett. B, 490, 27-35, 2000.

Boezio, M. et al., The cosmic-ray proton and helium spectra measured with the CAPRICE98 balloon experiment, Astropart. Phys., 19, 583604, 2003.

Briesmeister, J. F., MCNP-A general Monte Carlo N-particle transport code, version 4B, Rep. LA-12625-M, Los Alamos Natl. Lab., Los Alamos, N. M., 1997.

Carrier, W. D., Apollo drill core relationships, Moon, 10, 183-194, 1974.

Castagnoli, G. C. and D. Lal, Solar modulation effects in terrestrial production of carbon-14, Radiocarbon, 22(2), 133-158, 1980.

Dagge, G., P. Dragovitsch, D. Filges, and J. Bruckner, Monte Carlo simulation of Martian gamma-ray spectra induced by galactic cosmic rays, Proc. Lunar Planet. Sci. Conf., 21, 425-435, 1991.

Evans, L. G., R. C. Reedy, and J. I. Trombka, Introduction to planetary remote sensing gamma ray spectroscopy, in Remote Geochemical Analysis: Elemental and Mineralogical Composition, 167-198, Cambridge Univ. Press, New York, 1993.

Fasso, A., A. Ferrari, J. Ranft, and P. R. Sala, Proceedings of the IV International Conference on Calorimetry in High Energy Physics, La 
Biodola, Italy, 1993.

Feldman, W. C., W. V. Boynton, and D. M. Drake, Planetary neutron spectroscopy from orbit, in Remote Geochemical Analysis: Elemental and Mineralogical Composition, 213-234, Cambridge Univ. Press, New York, 1993.

Fukahori, T., Y. Watanabe, N. Yoshizawa, F. Maekawa, S. Meigo, C. Konno, N. Yamano, A. Yu. Konobeyev, and S. Chiba, JENDL high energy file, J. Nucl. Sci. Technol., Suppl., 2, 25-30, 2002.

Gasnault, O., C. d'Uston, W. C. Feldman, and S. Maurice, Lunar fast neutron leakage flux calculation and its elemental abundance dependence, J. Geophys. Res., E105, 4263-4271, 2000.

Gustafsson, K., L. Sihver, D. Mancusi, and T. Sato, PHITS simulations of the Matroshka experiment, Adv. Space Res., (submitted).

Hasebe, N., E. Shibamura, T. Miyachi, T. Takashima, M. Kobayashi, O. Okudaira, N. Yamashita, S. Kobayashi, T. Ishizaki, K. Sakurai, M. Miyajima, M. Fujii, K. Narasaki, S. Takai, K. Tsurumi, H. Kaneko, M. Nakazawa, K. Mori, O. Gasnault, S. Maurice, C. d'Uston, R. C. Reedy, and M. Grande, Gamma-ray spectrometer (GRS) for lunar polar orbiter SELENE, Earth Planets Space, 60, 299-312, 2008.

Hasebe, N., E. Shibamura, T. Miyzchi, T. Takashima, M. Kobayashi, O. Okudaira, N. Yamashita, S. Kobayashi, M. Hareyama, Y. Karouji, S. Kodaira, K. Sakurai, K. Iwabuchi, K. Hayatsu, S. Nemoto, M. Ebihara, T. Hihara, C. d'Uston, S. Maurice, O. Gasnault, B. Diez, and R. C. Reedy, First results of High Performance Germanium Gamma-Ray Spectrometer onboard Lunar Orbiter SELENE (KAGUYA), J. Phys. Soc. Jpn. Suppl., 78, 18-25, 2009.

Hayatsu, K., M. Hareyama, S. Kobayashi, N. Yamashita, M. Miyajima, K. Sakurai, and N. Hasebe, Radiation doses for human exposed to galactic cosmic rays and their secondary products on the lunar surface, Biol. Sci. Space, 22(2), 59-66, 2008.

Hayatsu, K., M. Hareyama, S. Kobayashi, N. Yamashita, K. Sakurai, and N. Hasebe, HZE particle and neutron dosages from cosmic rays on the lunar surface, J. Phys. Soc. Jpn. Suppl., 78, 149-152, 2009.

Ishibashi, K. et al., Measurement of neutron-production doubledifferential cross sections for nuclear spallation reaction induced by 0.8 , 1.5 and 3.0 GeV protons, J. Nucl. Sci. Tech., 34(6), 529-537, 1997.

Iwase, H., K. Niita, and T. Nakamura, Development of general purpose particle and heavy ion transport monte carlo code, J. Nucl. Sci. Tech., 39, $1142,2002$.

Lal, D., Theoretically expected variations in the terrestrial cosmic ray production rates of isotopes, in the Last Millenia, Proc. Int. Sch. Phys. Enrico Fermi, 95, 216-233, 1985.

Lawrence, D. J., S. Maurice, and W. C. Feldman, Gamma-ray measurements from Lunar Prospector: Time series data reduction for the Gamma-Ray Spectrometer, J. Geophys. Res., 109, E07S05, 2004.

Mancusi, D., L. Sihver, K. Gustafasson, C. L. Tessa, S. B. Guetersloh, C. J. Zeitlin, J. Miller, L. H. Heilbronn, K. Niita, T. Sato, H. Nakashima, T. Murakami, and Y. Iwata, PHITS - benchmark of partial chargechanging cross sections for intermediate mass systems, Nucl. Instr. Meth. B, 254, 30-38, 2007.

Masarik, J. and R. C. Reedy, Gamma-ray production and transport in Mars, J. Geophys. Res., 101, 18,891-18,912, 1996.

McKinney, G. W., D. L. Lawrence, T. H. Prettyman, R. C. Elphic, W. C. Feldman, and J. J. Hagerty, MCNPX benchmark for cosmic ray interactions with the Moon, J. Geophys. Res., 111, E06004, 2006.

Mitchell, J. K., W. D. Carrier III, N. C. Costes, W. N. Houston, R. F. Scott, and H. J. Hovland, Apollo 17 preliminary report, NASA Spec. Publ., SP-330, 8-1, 1974.

Nara, Y., N. Otuka, A. Ohnishi, K. Niita, and S. Chiba, Relativistic nuclear collision at AGS energies from $\mathrm{p}+\mathrm{Be}$ to $\mathrm{Au}+\mathrm{Au}$ with hadronic cascade model, Phys. Rev. C, 61, 024901, 2000.

Niita, K., S. Chiba, T. Maruyama, H. Takada, T. Fukahori, Y. Nakahara, and A. Iwamoto, Analysis of the (N, xN') reactions by quantum molecular dynamics plus statistical decay model, Phys. Rev. C, 52, 5, 1995.

Niita, K., S. Meigo, H. Takada, and Y. Ikeda, High energy particle transport code NMTC/JAM, Nucl. Instr. Meth. B, 184, 406, 2001.

Niita, K., T. Sato, H. Iwase, H. Nose, H. Nakashima, and L. Sihver, PHITS - a particle and heavy ion transport code system, Radiat. Measure., 41, 1080-1090, 2006.
Prael, R. E., Model cross-section calculations using LAHET, in Nuclear Data Evaluation Methodology, edited by C. L. Dunford, 525-534, World Sci., River Edge, N. J., 1993.

Prael, R. E. and H. Lichtenstein, User guide to LCS. The LAHET Code System, Rep. LA-UR-89-3014, 76, Los Alamos Natl. Lab., Los Alamos, N. M., 1989.

Reedy, R. C., private communication, 2009.

Reedy, R. C. and J. R. Arnold, Interaction of solar and galactic cosmic ray particles with the moon, J. Geophys. Res., 77, 537-555, 1972.

Sato, T. and K. Niita, Analytical functions to predict cosmic-ray neutron spectra in the atmosphere, Radiat. Res., 166, 544-555, 2006.

Sato, T., K. Niita, H. Iwase, H. Nakashima, Y. Yamaguchi, and L. Sihver, Applicability of particle and heavy ion transport code PHITS to the shielding design of spacecrafts, Radiat. Measure., 41, 1142-1146, 2006.

Sato, T., L. Sihver, K. Gustafsson, D. Mancusi, and Niita, Shielding design of spacecrafts using PHITS, Am. Nucl. Soc. Trans., 99, 592, 2008a.

Sato, T., H. Yasuda, K. Niita, A. Endo, and L. Sihver, Development of PARMA: PHITS based analytical radiation model in the atmosphere, Radiat. Res., 170, 244-259, 2008b.

Shapiro, M. M. and R. Silberberg, Heavy cosmic ray nuclei, Ann. Rev. Nucl. Part. Sci., 20, 323, 1970.

Shikaze, Y. et al., Measurements of $0.2-20 \mathrm{GeV} / \mathrm{n}$ cosmic-ray proton and helium spectra from 1997 through 2002 with the BESS spectrometer, Astropart. Phys., 28, 154-167, 2007.

Sihver, L., Transport calculations and accelerator experiments needed for radiation risk assessment in space, Z. Med. Phys., 18, 253-264, 2008.

Sihver, L. et al., Recent developments and benchmarking of the PHITS code, Adv. Space Res., 40, 1320-1331, 2007.

Sihver, L. et al., Benchmarking of calculated projectile fragmentation cross-sections using the 3-D, MC codes PHITS, FLUKA, HETCHEDS, MCNPX_HI, and NUCFRG2, Acta Astronaut., 63, 865-877, 2008a.

Sihver, L., D. Matthiä, T. Koi, and D. Mancusi, Dose calculations at high altitudes and in deep space with Geant4 using BIC and JQMD models for nucleus-nucleus reactions, New J. Phys., 10, 105019, 2008b.

Sihver, L., T. Sato, K. Gustafsson, V. A. Shurshakov, and G. Reitz, Simulations of the MTR-R and MTR Experiments at ISS, and Shielding Properties Using PHITS, Proc. 2009 IEEE Aerospace Conf., Big Sky, MT, 2009 (peer-reviewed).

Sihver, L., K. Gustafsson, D. Mancusi, T. Sato, K. Niita, H. Iwase, Y. Iwamoto, N. Matsuda, H. Nakashima, and Y. Sakamoto, An update of recent PHITS code, Adv. Space Res., (submitted).

Simpson, J. A., Elemental and isotopic composition of the galactic cosmic rays, Ann. Rev. Nucl. Part. Sci., 33, 323-381, 1983.

United Nations Scientific Committee on the Efffects of Atomic Radiation (UNSCEAR) report, http://www.unscear.org/docs/reports/1988/ 1988c_unscear.pdf, 1988.

Watanabe, Y. et al., T, Nuclear data evaluations for JENDL high-energy file, Proc. Int. Conf. Nucl. Data. Sci. Tech., 326-331, 2005.

Woolum, D. S., D. S. Burnett, and C. A. Bauman, Apollo 17 preliminary science report, NASA, Spec. Publ., SP-330, 18-1, 1973.

Woolum, D. S. and D. S. Burnett, In-situ measurement of the rate of ${ }^{235} \mathrm{U}$ fission induced by lunar neutrons, Earth Planet. Sci. Lett., 21, 153-163, 1974a.

Woolum, D. S. and D. S. Burnett, Lunar neutron capture rates and surface mixing of the regolith, Abstr. Lunar Planet. Sci. Conf., 5, 848-850, 1974b.

Woolum, D. S., D. S. Burnett, M. Furst, and J. R. Weiss, Measurement of the lunar neutron density profile, Moon, 12, 231-250, 1975.

Yamashita, N., N. Hasebe, T. Miyachi, M. Miyajima, K. Sakurai, M. Kobayashi, O. Okudaira, H. Yamamoto, E. Shibamura, Y. Uchihori, and M. Kase, Energy spectra of prompt gamma rays from Al and Fe thick targets irradiated by helium and proton beams: Concerning planetary gamma-ray spectroscopy, J. Phys. Soc. Jpn., 75, 054201, 2006.

S. Ota (e-mail: shuyaota@aoni.waseda.jp), S. Kobayashi, L. Sihver, N. Yamashita, and N. Hasebe 